\title{
Microtomography of the human tooth-alveolar bone complex
}

\author{
Michel Dalstra*a, Paolo M. Cattaneo ${ }^{\mathrm{a}}$, Felix Beckmann ${ }^{\mathrm{b}}$, Maurício T. Sakima ${ }^{\mathrm{c}}$, \\ Carsten Lemor ${ }^{\mathrm{a}}$, Morten G. Laursen ${ }^{\mathrm{a}}$, Birte Melsen ${ }^{\mathrm{a}}$ \\ ${ }^{a}$ Department of Orthodontics, School of Dentistry, University of Aarhus, \\ Vennelyst Boulevard 9, 8000 Aarhus C, Denmark \\ ${ }^{\mathrm{b}}$ GKSS Research Center Geesthacht, Max-Planck-Str. 1, 21502 Geesthacht, Germany \\ 'Department Clínica Infantil, University of São Paulo State, Araraquara, Brazil
}

\begin{abstract}
In this study the structure of the adult human dentoalveolar process is examined using conventional and synchrotron radiation-based microtomography (SR $\mu \mathrm{CT}$ ). Mandibular and maxillary segments containing two to five adjacent teeth were harvested at autopsy from 49 adult donors. These segments were embedded in blocks of methylmetacrylate and scanned using a conventional table-top $\mu$ CT-scanner at a pixel size and slice thickness of $35 \mu \mathrm{m}$. A few segments were also scanned at a synchrotron facility at an initial pixel size of $16.4 \mu \mathrm{m}$, which was binned by a factor 2 to result in an effective voxel size of almost $32.8 \mu \mathrm{m}$. The three-dimensional reconstructions revealed how intricately the teeth are supported by the alveolar bone. Furthermore, this support is highly inhomogeneous with respect to the buccal, mesial, lingual and distal quadrants. Reflecting their various degrees of mineralization, tissues like bone, dentine, enamel and cementum, could well be identified, especially in the scans made with SR $\mathrm{CCT}$. Despite comparable voxel sizes, the reconstructed data-sets obtained with conventional $\mu \mathrm{CT}$ were less detailed and somewhat fuzzy in appearance compared to the data-sets of SR $\mathrm{CCT}$. However, for quantification of macroscopical features like the thickness of the alveolar wall or the presence of dehiscences/fenestrations this seemed sufficient.
\end{abstract}

Keywords: microtomography $(\mu \mathrm{CT})$, synchrotron radiation, teeth, alveolar bone, dentoalveolar process

\section{INTRODUCTION}

In order to understand the load transfer from the tooth through the periodontal ligament (PDL) and the alveolar bone proper or lamina dura to the rest of the jaw, it is important to know the three-dimensional (3D) geometry and anatomy of the tooth-alveolar bone complex. Although conventional and recently also dental cone-beam CT-scanning have gained ground with regard to spatial resolution, microtomography is at present still the only means available to obtain detailed information about the spatial structure and architecture in the micrometer range. It should be realized, however, that this technique can only be used on ex-vivo human samples and not on actual patients. Originally this visualization modality was introduced in the field of medical science in the early 1990's to examine bone biopsies for osteoporosis by quantifying the trabecular network. ${ }^{1}$ Since then microtomography has become a well-established tool in bone research. Occasionally teeth and/or dental implants and their surrounding alveolar bone have been reported to have been $\mu C T$ scanned before, ${ }^{2-6}$ yet in most cases the material comes from animal studies, while microtomographic studies of the human dentoalveolar process are rare as a result of the limited availability of donor material.

As part of a larger on-going study to examine the structure of the dentoalveolar process and tooth migration patterns in an adult human population, the opportunity arose to $\mu$ CT-scan jaw segments, which had been obtained at autopsy at the Department of Forensic Medicine, University Hospital Aarhus, Denmark. In the first part of the study only mandibular segments with a lateral incisor, canine and first premolar were harvested due to the relative ease of excision. In the second part of the study, also maxillary segments and mandibular segments with posterior teeth were collected, so that elements from the entire dentition were represented.

*mdalstra@odont.au.dk; phone (+45) 8942 4190; fax (+45) 8619 2752; www.odont.au.dk 
Having local access to a conventional table-top $\mu$ CT-scanner, all segments would be scanned with this apparatus. A handful of segments were selected to be scanned at HASYLAB/DESY synchrotron facility in Hamburg, Germany as well. However, due to the limited amount of allocated beamtime and major reconstruction work at beamline W2 of the DORIS ring at HASYLAB/DESY only three segments have thus been scanned so far.

State-of-the-art synchrotron radiation-based $\mu \mathrm{CT}$ (SR $\mu \mathrm{CT}$ ) allows for spatial resolutions in the order of around 300$1,000 \mathrm{~nm}$ and, perhaps even more importantly, it strongly reduces image artifacts caused by magnification of a diverging beam and beam hardening, which have a substantial influence on the overall image quality of microtomographs taken with a conventional X-ray source. As synchrotron radiation is monochromatized, beam hardening in fact plays no role at all and the gray values in the reconstructed scan images are directly related to the local linear attenuation coefficient of the scanned material, which in case of mineralized biological tissues means that a direct relationship with the local degree of mineralization exists. Actually, SR $\mu \mathrm{CT}$ is so specific that not only different types of tissues with various degrees of mineralization can be separately identified, but also minor changes in the mineralization patterns within the same type of tissue can be detected. ${ }^{7-12}$ The various dentoalveolar calcified tissues, like bone, dentine, enamel and cementum, display a wide range in degrees of mineralization (from 60 to 98 percent). The forte of SR $\mu C T$ - scans would therefore be to better distinguish between these different tissues, as due to the size of the excised segments, it was understood from the beginning that the spatial resolution for the SR $\mu \mathrm{CT}$ - scans would not be significantly better than what can be achieved with a $\mu \mathrm{CT}$-scanner with a conventional X-ray source.

It was the aim of this study to assess the three-dimensional mesoscopic structure of the human dentoalveolar process and compare the efficacy of conventional $\mu \mathrm{CT}$ and SR $\mu \mathrm{CT}$ being used for this purpose.

\section{MATERIAL \& METHODS}

\subsection{Material}

Segments from both the human mandibular and maxillary dentoalveolar process, containing between two and five adjacent teeth, were obtained at autopsy at the Department of Forensic Medicine, University Hospital Aarhus, Denmark from a total of 49 donors - 32 males and 17 females - ranging between 19 and 55 years of age with the consent of the Ethical Committee of the University of Aarhus. Upon excision, usually care was taken not to include teeth with amalgam fillings, although this could not be guaranteed in all cases.

The segments were fixated in 70 percent alcohol prior to the embedding procedure. To minimize the total volume which had to be embedded, the coronal halves of the crowns were removed with a precision band saw (Exakt Apparatebau, Norderstedt, Germany). The embedding procedure was preceded by sequential dehydration in 96 and 99 percent alcohol and finally in an equal solution of 99 percent alcohol and acetone for 4 days each. The segments were then infiltrated by methylmetacrylate (MMA) monomer inside a vacuum pump for 1 week, followed by the final polymerization.

Finally, the resulting blocks containing the embedded segments were trimmed down to fit in the largest sample holder (an $80 \mathrm{~mm}$ long cylinder with a diameter of $37 \mathrm{~mm}$ ) of the conventional $\mu \mathrm{CT}$-scanner using the abovementioned band saw.

\subsection{Conventional microtomography}

All embedded segments were scanned at an energy of $70 \mathrm{kVp}$ using a table-top $\mu \mathrm{CT}$ system (Scanco Medical $\mu \mathrm{CT} 40$, Bassersdorf, Switzerland) in standard resolution mode with a pixel size and slice thickness of $35 \mu \mathrm{m}$. Most segments were scanned with the teeth's longitudinal axis perpendicular to the scan direction. Depending on the varying heights of the individual specimens, it generally required between 600 and 700 scans to cover the various tissue blocks' heights. Some segments proved too long to fit the sample holder's diameter. These segments were then scanned along their length instead and usually would require around 800 scans to cover the entire length. After scanning, the single-slice images were exported as 1,024x1,024 pixels TIFF-files (Tag Image File Format) for further processing. 


\subsection{Synchrotron radiation-based microtomography}

Due to limited accessibility to a SR $\mu C T$-system, only three of the segments could be scanned using this technique (while at present still two more segments are in the pipeline to be scanned). These segments were scanned with a SR-based microtomograph operating in absorption mode. ${ }^{13}$ The experiments were conducted at the wiggler beamline W2 of the DORIS ring at HASYLAB/DESY (Hamburg, Germany). This beamline operates at a photon energy range of 20 to 60 $\mathrm{keV}$ and has a beam size of $15 \times 4 \mathrm{~mm}^{2}$ and a best achievable spatial resolution of $3 \mu \mathrm{m}$. A double crystal Si(111) monochromator is used to monochromatize the incident white beam. After entering the X-ray detector, the monochromatic beam is converted by a fluorescent screen $\left(\mathrm{CdWO}_{4}, 500 \mu \mathrm{m}\right.$ thick) into visible light, which is finally projected on the CCD camera (Apogee KX2, Kodak KAF 1600, 1,536x1,024 pixels, pixel size $9 \mu \mathrm{m}$ ).

The three segments were scanned at a photon energy of $45 \mathrm{keV}$. Due to the size of these segments (about $20 \times 23 \times 29$ $\mathrm{mm}^{3}$ ), the best achievable pixel size was $16.4 \mu \mathrm{m}$. As the width of the samples exceeded the readout area of the CCD camera by almost a factor 2, the sample holder had to rotate from -180 to 180 degrees in steps of 0.25 degrees in order to acquire the full image with the rotation center located to the side of the CCD readout area. The two by 180 degrees shifted projection images were finally digitally combined into a complete projection image of almost the double CCD width. Due to the limited height of the beam, the segments had to be scanned at eight different, slightly overlapping heights.

\subsection{Postprocessing and visualization}

For the SR $\mu \mathrm{CT}$ scanning the single-slice reconstructions were calculated from the raw data (sinograms) with a filtered back-projection algorithm using IDL software (Research Systems Inc., Colorado). These images were subsequently stacked to create 3D voxel data-sets associated with a scanning session for one particular height. The data-sets of the eight individual heights were then merged to create data-sets of the entire segments. In order to both reduce noise and keep the amount of data to a manageable level, the data-sets were finally binned with a factor 2, so that their effective pixel size became $32.8 \mu \mathrm{m}$ and thereby comparable to those which had been scanned by conventional $\mu \mathrm{CT}$.

Special image processing software (VGStudio MAX, Volume Graphics GmbH, Heidelberg, Germany) was used to visualize the voxel data-sets of the SR-based microtomography and the stacked TIFF-files of the conventional microtomography. By using a transparency function with cut-off threshold values on the histogram of gray values, the voxels with the highest gray values become opaque, while the voxels with the lowest gray values become transparent. For the visualization of the non-mineralized tissues the inverse of this transparency function was employed in combination with false-coloring for the enhancement of these various phases and structures.

Macroscopical measurements of the thickness of the buccal and lingual alveolar wall as a function of the root length were performed for each tooth in the scanned data-sets. Simultaneously, the presence of any dehiscences and fenestrations was registered. No final results of these measurements will be reported here, as only parts of these measurements have been completed so far.

\section{RESULTS}

The single-slice images clearly display the various mineralized tissues. The two alveolar walls consist of dense cortical bone, whereas the core of the jaw bones consists of trabecular bone, which connects both the alveolar walls and the alveolar bone proper, which acts as the containment and support for the roots of the teeth. The "empty" space between the roots and the alveolar bone proper denotes the presence of the PDL. Within the roots, predominantly made up of primary dentine, two darker gradations of gray values can be observed, representing tissues with a slightly lower degree of mineralization. One is seen along the outer surface of the roots and this is the cementum layer into with the fibers of the PDL (Sharpey's fibers) are anchored into tooth surface. The other is seen along the root canals and pulp chambers and represents secondary dentine, a less matured - and therefore less mineralized - form of primary dentine.

Comparison of the single-slice images of the sample segments, which had been scanned with both conventional and SRbased $\mu \mathrm{CT}$ showed that the conventional $\mu \mathrm{CT}$ images were considerably less distinctive for the various mineralized tissues. Only the enamel on the remains of the crowns stood out clearly, but there were almost no gray value differences 
between the bone, the dentine and the cementum. The SR $\mu \mathrm{CT}$ single-slice images on the other hand did not only display a significant difference between these tissues, but also within these tissues slight gray value differences pointed to regions with different degrees of mineralization (Figure 1). Especially within the alveolar walls the circumferentially orientated haversian systems with the osteons and the haversian canals can be made out. Occasionally darker osteons are seen, which represent newly formed osteons, which have not fully matured yet.

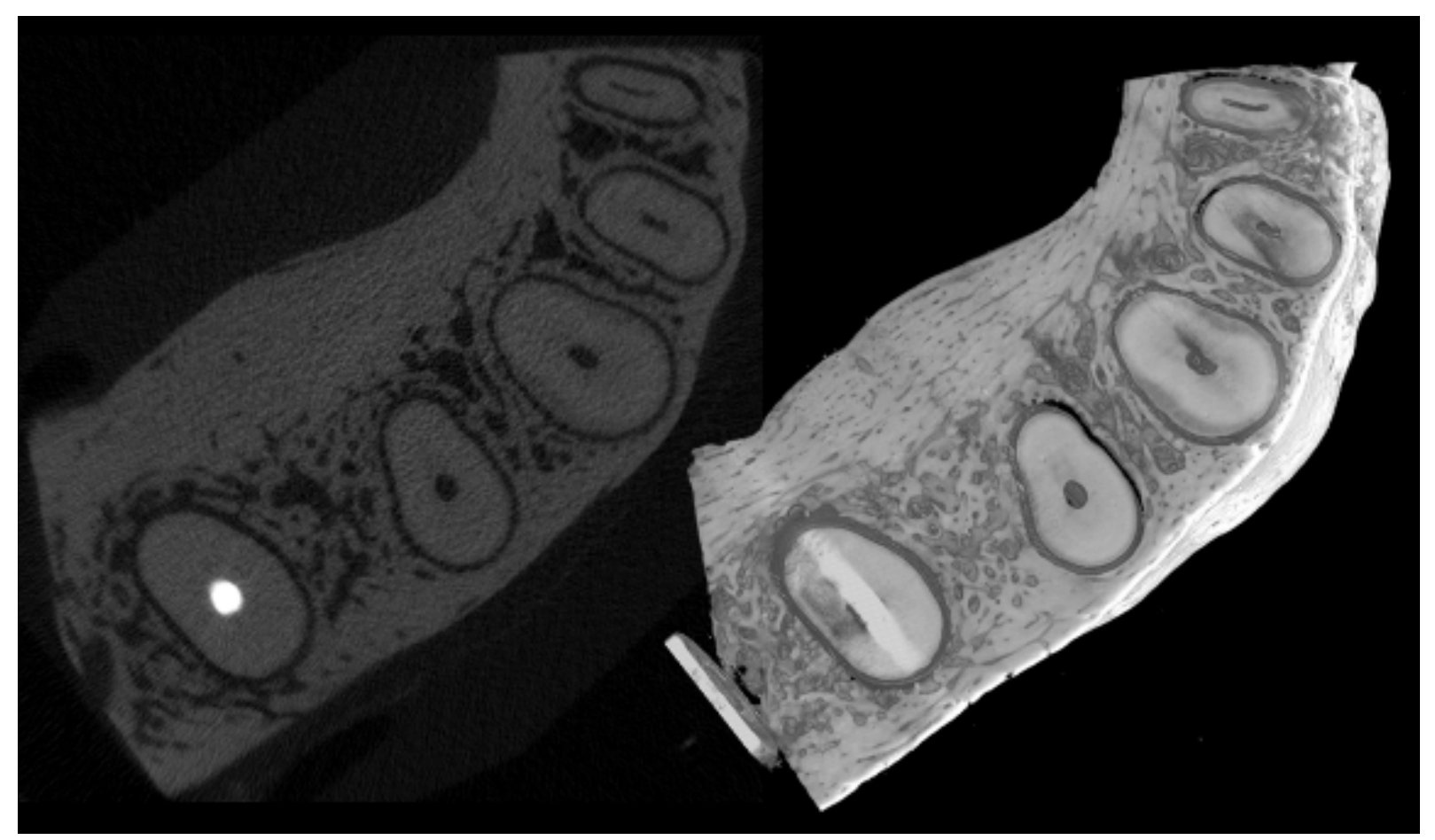

Fig. 1. A single-slice image of a mandibular jaw segment at mid-root level from a 32 year old male donor containing two incisors, a canine and two premolars, obtained with conventional $\mu \mathrm{CT}$ (left) and the corresponding slice from the 3D $\mathrm{SR} \mu \mathrm{CT}$ data-set (right). The amount of detail and the variety of the gray values in the latter is striking. Notice the amalgam-based filling of the root canal in the second premolar. The energy used for the SR $\mu$ CT had apparently not been sufficiently high enough to reconstruct this feature correctly, thereby leaving a smear-like artifact in the original scan direction. The bright disc-like object at the bottom left of the SR $\mu$ CT data-set is part of the cylindrical steel sample holder.

Comparison of the 3D reconstructions of the data-sets reflected the findings observed above for the single-slice images. The reconstructions based on conventional $\mu \mathrm{CT}$ appeared fuzzier and less detailed, even though the voxel sizes of these data-sets were almost the same as those for the SR $\mu \mathrm{CT}$ data-sets (Figure 2). The noise in the gray values in the roots of the teeth is substantial in the reconstructions based on conventional $\mu \mathrm{CT}$, making it more difficult to distinguish the cementum and secondary dentine from the primary dentine. Although the individual trabeculae in the core of the segments scanned with conventional $\mu \mathrm{CT}$ can well be identified, the general appearance of the trabecular bone is flat and the edges of the trabeculae are not sharp. This also applies to the visualization of the outside surface of the alveolar wall, where the SR $\mu \mathrm{CT}$ data-sets display much more detail with discontinuities of the cortical bone, entry and exit holes for blood vessels and nerves and imprints of blood vessels onto the surface. However, in the 3D reconstructions of both scanning modalities, the space of the PDL can be visualized equally well. 


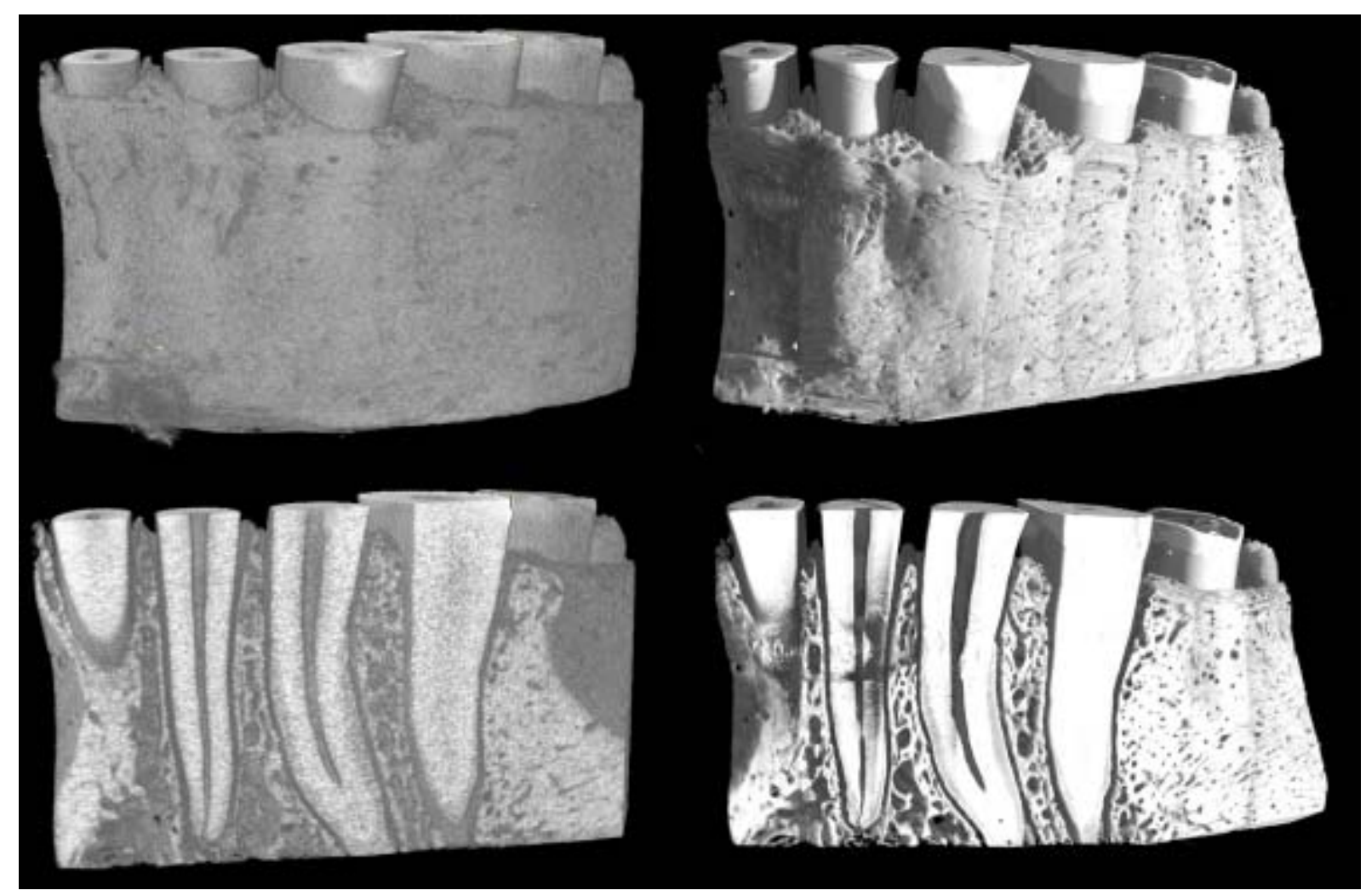

Fig. 2. A 3D reconstruction of the same mandibular jaw segment as seen in Figure 1. Again the data-set obtained with conventional $\mu \mathrm{CT}$ is shown on the left and the corresponding data-set obtained with SR $\mu \mathrm{CT}$ on the right. The bottom images represent longitudinal cuts through the middle of the roots of the lateral incisor and the canine. Notice the curvature of the root of the latter. Also notice a reconstruction artifact at mid-root level of the lateral incisor in the $\mathrm{SR} \mu \mathrm{CT}$ data-set (bottom right).

The 3D reconstructions of the segments allow for cross-sectional cuts through the segments in any arbitrary direction to be made (Figure 3). These cut-through views illustrate excellently how the teeth are supported by the alveolar bone. The suspending PDLs of the various teeth appear to have a fairly constant thickness of about 0.3 mm. Any irregularities and/or malformations in the outer shape of the roots are closely followed by corresponding adaptations in the shape of the alveolar bone proper or lamina dura, thus ensuring this uniform thickness of the PDL.

With the exception of their cervical part, the lamina durae have thicknesses, which are comparable to those of the PDLs. For all teeth the thickness of their lamina dura seems to decrease slightly towards the apex of the root. Cervically, the lamina durae widen and merge with the cortex, and here the cortical bone is the most porous. The apical two-thirds of the lamina dura are only loosely supported by an intricate network of trabeculae, which interconnect with the inner and outer cortices and/or the neighboring laminae durae. In particular towards the apices of the roots, this network becomes very porous.

Overall, the degree of mechanical support for the roots is rather inhomogeneous with regards to the anatomical position and direction along the roots. With the presence of the thick buccal and lingual alveolar walls, the roots are more firmly supported in this plane than in the mesiodistal direction. It should also be noted that the lingual alveolar wall is considerable thicker than on the buccal side, where for most teeth the alveolar wall is less than 1 mm thick for up to 60 percent of the root's length. Furthermore, the roots receive more support in the cervical region than in the apical region, where the trabecular supporting structures are very sparse indeed. 


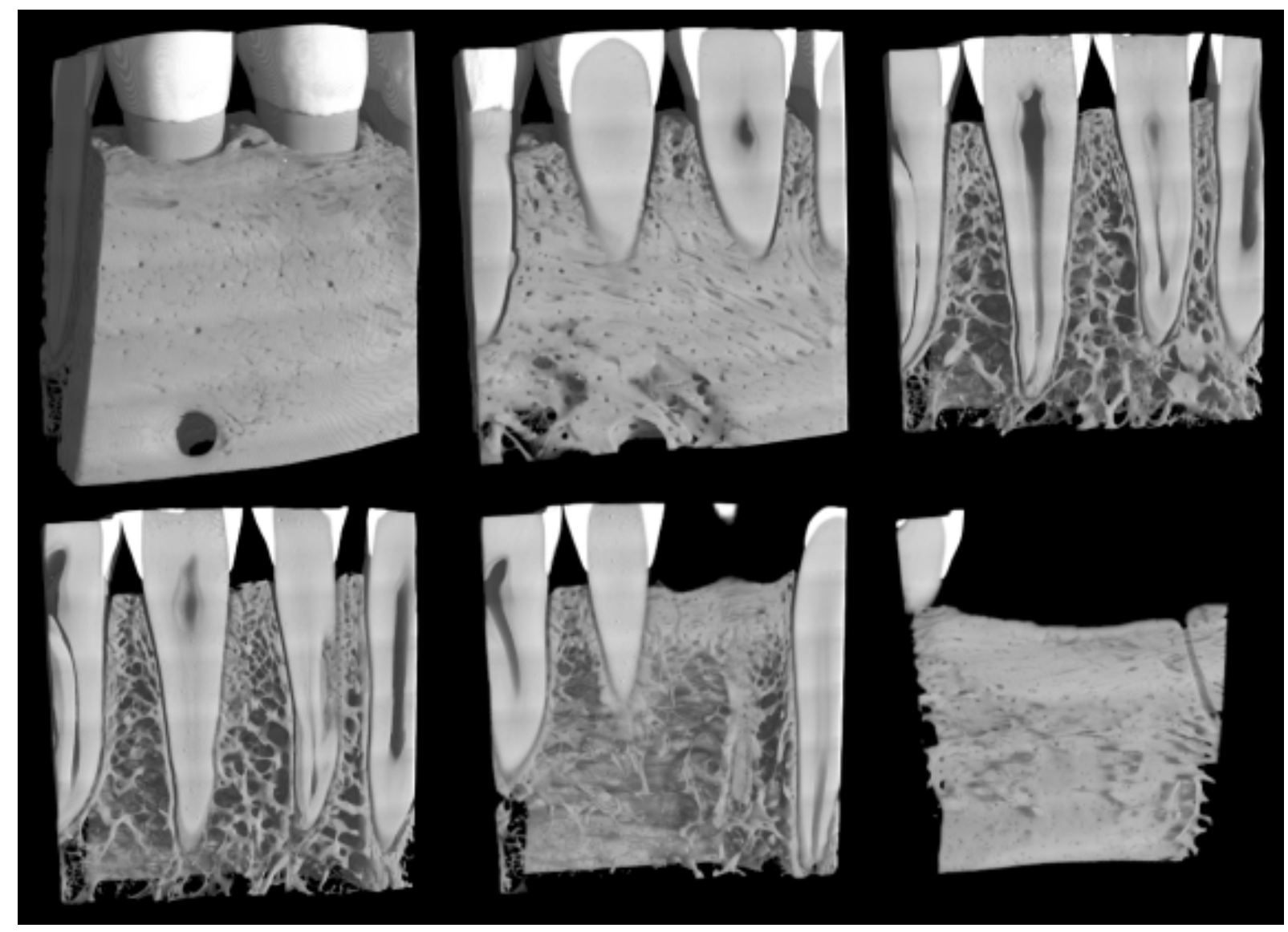

Fig. 3. A 3D reconstruction from an SR $\mu \mathrm{CT}$ data-set of a mandibular jaw segment of a 19 year old male donor with part of the canine, the two premolars and the mesial half of the first molar (top left). Going from left to right and from top to bottom, a series of sequential sagittal cut-through views are shown. Notice the slightly darker secondary dentine around the roots' pulp chambers. Also notice the growth disturbance along the root of the first premolar in the bottom left view.

The structure of the soft tissues could indirectly be visualized by displaying the MMA phase in the 3D data-sets. A longitudinal cross-section through the middle of the segment revealed that the embedding process had actually left quite a few air bubbles in the sample, especially in the more porous part of the alveolar bone towards the bottom of the segment (Figure 4). The impression of the MMA does however reveal an amazing network of blood vessels, marrow cavities, the PDLs, the pulp chambers and the root canals. The haversian canals in the cortical bone are predominantly oriented in the mesiodistal direction and form an extensive network with many branches veering off into the bone marrow and the PDLs. This can best be appreciated in a detailed view of a small section of the segment showing the transitional area of the outer cortex, lamina dura and trabecular core (Figure 5). Also here some immature (darker) osteons following the circumferential orientation can be seen.

\section{CONCLUSION}

Microtomography provides a novel way to understand the 3D structure of the human dentoalveolar complex. Seeing the interconnecting networks of trabeculae and soft tissues makes one realize how intricately the teeth are supported in the 
jaws. Especially in the orthodontic community there is a general belief that teeth are supported uniformly by the alveolar bone. However, the present study shows this to be untrue, as the mechanical support of the teeth is in fact strongly dependent on the anatomical directions and the level of the root length.

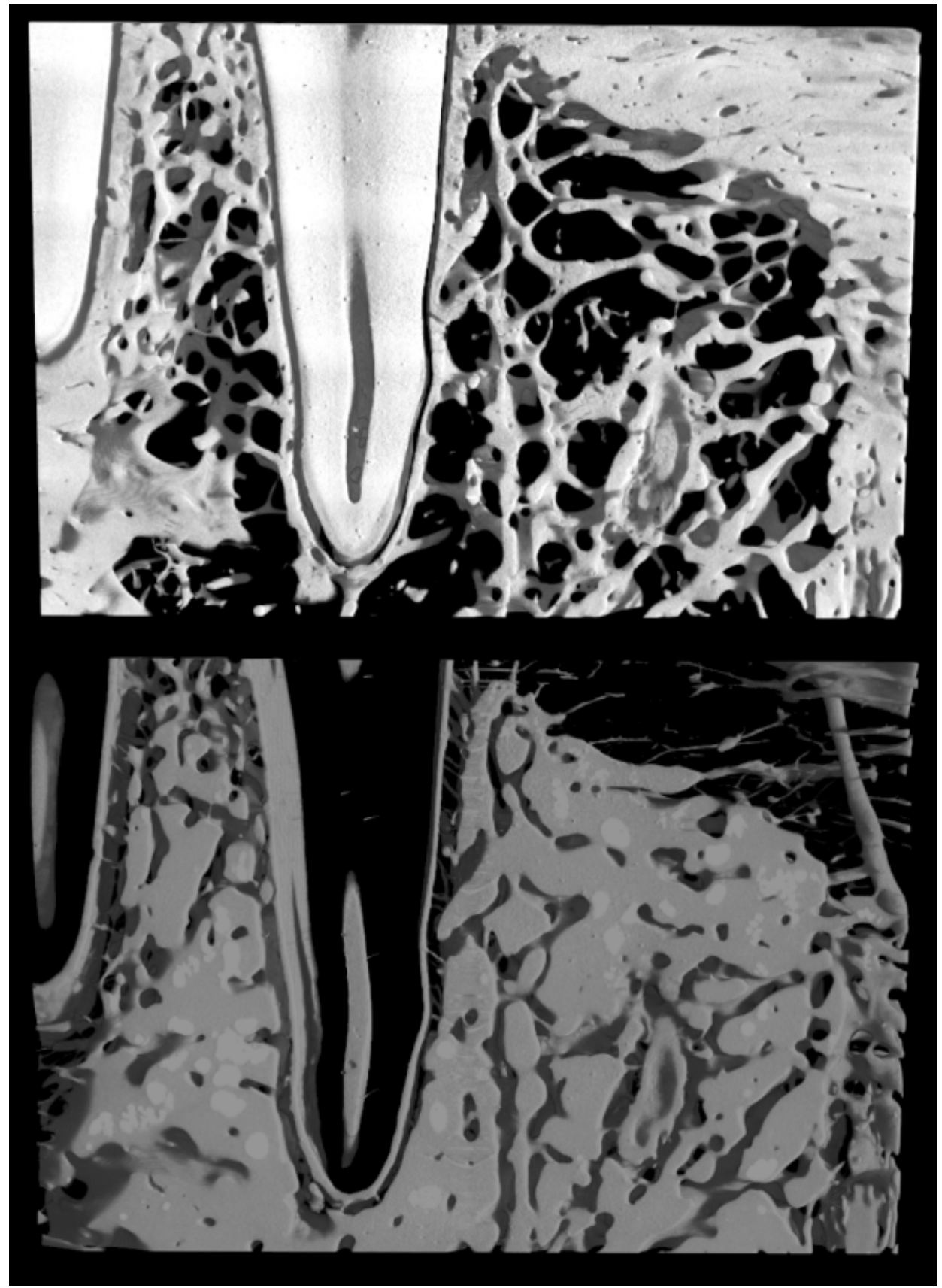

Fig. 4. Mesiodistal cross section through the second premolar and the first molar from the 3D reconstruction shown in Figure 3. The top image displays the mineralized tissues, while the bottom image displays the complementary materials (MMA and air) in the data-set, representing the original soft tissues. Notice the root canals and the periodontal ligaments and how the latter are connected by thin blood vessels to the bone marrow running through the alveolar bone proper. Also notice the large blood vessel transversing the cortical alveolar wall on the right. 


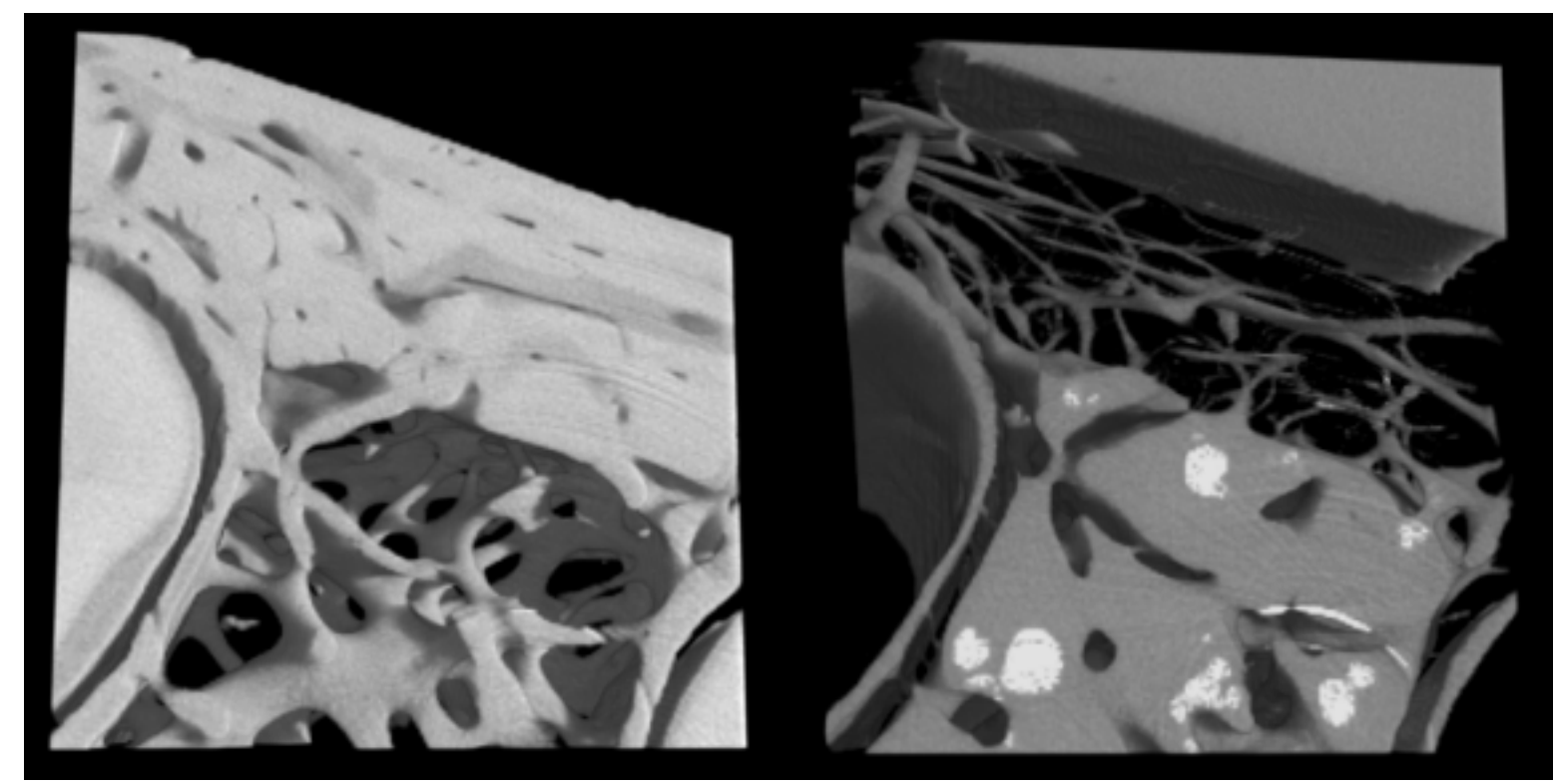

Fig. 5. Detailed transverse section at mid-root level from the 3D reconstruction shown in Figure 3. The left image displays the mineralized tissues, and in particular the cementum layer on the outside of the root and osteons with different degrees of mineralization in the alveolar wall can clearly be observed. The right image displays the complementary tissues in the data-set with the periodontal ligament and the network of haversian canals in the alveolar wall. The triangular structure on the top is the MMA embedding material on the outside of the sample, while the bright areas represent air bubbles, where the MMA did not achieve full penetration.

For the quantification and/or measurements of most macro- and mesoscopical features of dentoalveolar segments conventional $\mu \mathrm{CT}$ is sufficient. For visualization purposes, SR $\mu \mathrm{CT}$ is clearly the method of choice as, due to its monochromatic beam, this technique is more able to distinguish between tissues with slightly different degrees of mineralization. Limited accessibility of this scanning method, however, prevents the scanning of larger series.

\section{ACKNOWLEDGMENTS}

This work has been financially supported by The European Commission (IHP-contracts II-00-064 EC \& II-03-052 EC), the Danish Research Council for Natural Sciences (DANSYNC program) and the Aarhus University Research Fund (E2004-SUN-1-120).

\section{REFERENCES}

1. R. Müller, T. Hildebrand and P. Rüegsegger, "Non-invasive bone biopsy: a new method to analyse and display the three-dimensional structure of trabecular bone”, Phys Med Biol, 39, 145-164, 1994.

2. C. Verna, M. Dalstra and B. Melsen, "The rate and the type of orthodontic tooth movement is influenced by bone turnover in a rat model”, Eur J Orthod, 22, 343-352, 2000.

3. C. Verna, M. Dalstra, T.C. Lee, P.M. Cattaneo and B. Melsen, "Microcracks in the alveolar bone following orthodontic tooth movement: a morphological and morphometric study", Eur J Orthod, 26, 459-467, 2004.

4. G. Yip, P. Schneider and E.W. Roberts, "Micro-computed tomography: high resolution imaging of bone and implants in three dimensions”, Sem Orthod, 10, 174-187, 2004. 
5. P.M. Cattaneo, M. Dalstra, F. Beckmann, T. Donath and B. Melsen, "Comparison of conventional and synchrotron radiation based microtomography of bone around dental implants”, In: U. Bonse (Ed.) Developments in X-Ray Tomography IV, Proceedings of SPIE Vol. 5535, Bellingham WA: SPIE, pp. 757-764, 2004.

6. M. Dalstra, P.M. Cattaneo and F. Beckmann, "Three-dimensional structure of the dentoalveolar process studied with synchrotron radiation-based microtomography”, In. Z. Davidovitch, J. Mah and S. Suthanarak (Eds.) Biological Mechanisms of Tooth Eruption, Resorption and Movement, Harvard Society for the Advancement of Orthodontics, Boston MA, 2006.

7. S. Nuzzo, F. Peyrin, P. Cloetens, J. Baruchel and G. Boivin, "Quantification of the degree of mineralization of bone in three dimensions using synchrotron radiation microtomography”, Med Phys, 29, 2672-2681, 2002.

8. S. Nuzzo, M.H. Lafage-Proust, E. Martin-Badosa, G. Boivin, T. Thomas, C. Alexandre and F. Peyrin, "Synchrotron radiation microtomography allows the analysis of three-dimensional microarchitecture and degree of mineralization of human iliac crest biopsy specimens: effects of etidronate treatment”, J Bone Miner Res, 17, 1372-1382, 2002.

9. M. Dalstra, E. Karaj, F. Beckmann, T. Andersen and P.M. Cattaneo, "Osteonal mineralization patterns in cortical bone studied by synchrotron radiation-based computed microtomography and scanning acoustic microscopy”, In: U. Bonse (Ed.) Developments in X-Ray Tomography IV, Proceedings of SPIE Vol. 5535. Bellingham, WA: SPIE, pp. 143-151, 2004.

10. S.E.P. Dowker, J.C. Elliot, G.R. Davis, R.M. Wilson and P. Cloetens, "Synchrotron x-ray microtomographic investigation of mineral concentrations at micrometre scale in sound and carious enamel”, Caries Res, 38, 514-522, 2004.

11. J.H. Kinney, R.K. Nalla, J.A. Pople, T.M. Breunig and R.O. Ritchie, “Age-related transparent root dentin: mineral concentration, crystallite size, and mechanical properties”, Biomat, 26, 3363-3376, 2005.

12. O. Prymak, H. Tiemann, I. Sötje, J.C. Marxen, A. Klocke, B. Kahl-Nieke, F. Beckmann, T. Donath and M. Epple, "Application of synchrotron-radiation-based computer microtomography (SRmuCT) to selected biominerals: embryonic snails, statoliths of medusae, and human teeth”, J Biol Inorg Chem, 27, 1-8, 2005.

13. U. Bonse, F. Busch, O. Günnewig, F. Beckmann, R. Pahl, G. Delling, M. Hahn and W. Graeff, “3D computed X-ray tomography of human cancellous bone at 8 microns spatial and $10^{-4}$ energy resolution”, Bone Min, 25, 25-38, 1994. 